\begin{tabular}{|c|c|c|}
\hline & Int.J.Curr.Microbiol.App.Sci (2021) 10(10): 319-325 & \\
\hline & $\begin{array}{l}\text { International Journal of Current Microbiology and Applied Sciences } \\
\text { ISSN: 2319-7706 Volume } 10 \text { Number } \mathbf{1 0}(\mathbf{2 0 2 1 )} \\
\text { Journal homepage: } \underline{\text { http://www.ijcmas.com }}\end{array}$ & 39 \\
\hline $\begin{array}{l}\text { EXCELLENT } \\
\text { PUBLISHERS }\end{array}$ & & \\
\hline
\end{tabular}

\title{
Antibiogram of Staphylococcus aureus in a Tertiary Care Hospital: Changing Trends
}

\author{
Priyanka Pradhan $^{1 *}$, Sneha Mohan ${ }^{2}$, Tarana Sarwat ${ }^{2}$ and Dalip Kakru ${ }^{2}$ \\ ${ }^{1}$ Department of Medical Microbiology, ${ }^{2}$ Department of Microbiology, \\ Sharda University, India \\ *Corresponding author
}

Methicillin resistant Staphylococcus aureus (MRSA) is major cause of nosocomial and community infections. Its prevalence varies with country and with hospitals within a country. Therefore, it is important for continuous surveillance in hospitals and other healthcare facilities in order to limit the spread of infections caused by MRSA. To determine the antibiogram of Staphylococcus aureus in a tertiary care

Keywords

MRSA, MSSA,

S. aureus, mecA gene

Article Info

Accepted: 15 September 2021 Available Online: 10 October 2021 hospital and to determine the change in trends in the antimicrobial susceptibility pattern of Staphylococcus aureus. To determine the prevalence of MRSA in a tertiary care hospital. This cross- sectional study was conducted at the Department of Microbiology, SMSR, Sharda University and Hospital. A total of 100 strains identified by morphological and biochemical characteristics were tested for antibiotic susceptibility using Kirby-Bauer disc diffusion method and the Prevalence of Inducible Clindamycin Resistance among the isolates. 100 out of 13,639 isolates clinical comprising samples were obtained Pus (78.6\%), Blood (7\%), Swab (4.1\%), Sputum (4.1\%), Urine (4.1\%), Semen (2\%). Maximum MRSA were obtained from pus samples $(81 \%)$. However, out of total 7 isolates of Staphylococcus aureus obtained from blood 4 were MRSA and 3 were MSSA. The prevalence of MRSA that is (40\%) infections was a high in our setup and is comparable to studies done earlier. This trend is particularly alarming for Staphylococcus aureus because of the severity and diversity of disease caused by this uniquely versatile pathogen.

\section{Introduction}

Staphylococcus aureusis a Gram-positive coccus which belongs to the family Micrococcaceae and is a facultative anaerobe that can grow without the need for oxygen. It is a usual member of the microbiota of the body, frequently found in the upper respiratory tract and on the skin ${ }^{(1)}$. They are round cells, approximately $1 \mu \mathrm{m}$ in diameter form grape like (Greek-staphyle) clusters indicative of the ability to divide in more than one plane. They are non-motile, non-spore forming. An estimated $20 \%$ to $30 \%$ of the 
human population are long term carriers of $S$. aureus which can be found as part of the normal skin flora, in the nostrils ${ }^{(2,3)}$ and as a normal inhabitant of the lower reproductive tract of women ${ }^{(2,4)}$. Although $S$. aureus acts as a commensal of the human microbiota it can also become an opportunistic pathogen, being a common cause of skin infections including abscesses, respiratory infections such as sinusitis, bone infections such as osteomyelitis and food poisoning. S. aureus has become a worldwide problem in clinical medicine because of its resistance towards antibiotics. Methicillin was first introduced in 1959 and was very effective in treating patients with penicillin-resistant Staphylococcus aureus infection but eventually resistance to methicillin also emerged ${ }^{(2)}$.

\section{Materials and Methods}

It was a cross-sectional study conducted at the Department of Microbiology and Sharda Hospital from December 2019 - November 2020. A total number of $100 \mathrm{~S}$. aureus isolates were collected from different clinical samples from outpatients and in patients visiting and admitted to the Sharda Hospital, a 900-bed tertiary care hospital in Greater Noida.

\section{Inclusion criteria}

All Staphylococcus aureus strains isolated from a clinical sample received in the central laboratory.

\section{Exclusion criteria}

All isolated strains other than Staphylococcus aureus.

\section{Isolation and Identification of Staphylococcus aureus isolates}

The various specimens such as urine, blood, pus, sputum, swabs, catheter tips, pleural fluid obtained from patient admitted in hospital were submitted in the Microbiology Laboratory from IPD and OPD patients from the bacteriological analysis were included in this study. All the specimens were inoculated onto Blood agar and MacConkey agar (HiMedia, India). Urine specimens were inoculated CLED agar (Hi-Media, India). Isolates of S.aureus were identified by colony morphology, Gram staining, Catalase test, Coagulase test, Cultured in selective media in Mannitol salt agar.

\section{Disc diffusion test by Kirby-Bauer method}

These isolates were further tested for antimicrobial susceptibility to different antibiotics like Levofloxacin $(5 \mu \mathrm{g})$, Cefoxitin $(30 \mu \mathrm{g}), \quad$ Clindamycin $(2 \mu \mathrm{g}), \quad$ Vancomycin $(30 \mu \mathrm{g})$, Gentamicin $(10 \mu \mathrm{g} 0$, Tetracycline $(30 \mu g)$, Ciprofloxacin $(5 \mu \mathrm{g})$, Erythromycin $(15 \mu \mathrm{g})$, Linezolid $(30 \mu \mathrm{g})$, Penicillin (10 units), Teicoplanin $(30 \mu \mathrm{g})$ by Kirby Bauer disc diffusion method after matching bacterial suspension with Mac Farland's 0.5 standards according to CLSI guidelines 2020 using standard microbiological techniques on Mueller Hinton agar plates Zone of inhibition equal to or less than $6 \mathrm{~mm}$ for broth. After 24 hours of incubation at $37^{\circ} \mathrm{C}$, all plates were read according to standard procedure.

\section{Detection of inducible Clindamycin resistant}

It is used to detect inducible clindamycin resistance in staphylococci.

It was detected by the disc diffusion method using Clindamycin and Erythromycin discs.

D test was performed by disc diffusion, placing a $15 \mu \mathrm{g}$ Erythromycin disk in proximity to a $2 \mu \mathrm{g}$ clindamycin disk on an agar plate that has been inoculated with staphylococci isolate; the plate is then incubated overnight. 
A flattening of the zone of inhibition around the clindamycin disk proximal to the Erythromycin disk (producing a zone of inhibition shaped like the letter D) is considered a positive result and indicates that the Erythromycin has induced Clindamycin resistance (a positive "D-zone test").

\section{Results and Discussion}

The present study was conducted at the department of Microbiology, School of Medical Sciences and Research, Sharda University, Sharda Hospital, Greater Noida, from $1^{\text {st }}$ December to $30^{\text {th }}$ November 2020. The total no of 100 strains of Staphylococcus aureus were isolated from patients admitted\& attending the OPD at Sharda University during the study period. The table given below depicts the no. of patient's sample received in the bacteriology laboratory for culture \& sensitivity during the study period.

\section{Sample distribution}

Out of 100 isolates the Staphylococcus aureus were obtained from following clinical samples in order of frequency: Pus (78.6\%), Blood (7\%), Swab (4.1\%), Sputum (4.1\%), Urine (4.1\%), Semen (2\%). Maximum MRSA were obtained from pus samples $(81 \%)$. However, out of total 7 isolates of Staphylococcus aureus obtained from blood 4 were MRSA and 3 were MSSA.

\section{Age distribution}

Among the isolates of Staphylococcus aureus studied $(n=100)$ which were obtained from the age group of 41 to 50 years. Prevalence of Staphylococcus aureus infection was found to be less in extremes of age group that is $>80$ years (0). The prevalence of MRSA infection was also found to be higher among the age group of 41 to 50 that is $(20 \%)$.

\section{Sex distribution}

The maximum no of isolates of Staphylococcus aureus from the clinical samples were isolated from males that is 63 and 37 were females. MRSA infections were found to be more prevalent in males (63\%) than in females $(37 \%)$.

The present study was conducted for the prevalence of methicillin resistant $S$. aureus infection in our hospital and to know the antibiotic susceptibility profiles and resistance patterns. In our study 868 bacterial isolates cultured from various $(13,639)$ clinical specimens over a period of 12 months, 100 $(0.73 \%)$. This finding was correlated well with the study conducted by Grundmann et al., (2010) where all the overall percentage of infection caused by Staphyloccoccus aureus was $61.7 \%{ }^{(6)}$. Isolates were identified as Staphylococcus aureus in which $40 \%$ were MRSA and $60 \%$ were MSSA as compared to study Silvana M.M et al., (2005) ${ }^{(7)}$. In our study, the total isolates of Stapylococcus aureus were 100 from pus (78.6\%), blood $(7 \%)$, urine $(4.1 \%)$, swab $(4.1 \%)$, semen $(2 \%)$, sputum $(4.1 \%)$ in which the maximum number of MRSA isolates were obtained from pus samples $(81 \%)$ shown in table no 2 . These findings were similar to a study conducted by Kulkarni et al., (2014) who reported the higher percentage $(82.38 \%)$ of MRSA isolates from pus samples. Another similar study by Anupurba et al., reported $76 \%$ MRSA isolate from urine, $56.5 \%$ from sputum/throat swab, $52.5 \%$ from pus and wound swab and $49.1 \%$ from blood ${ }^{(8)}$. The prevalence of MRSA in the present study showed $40 \%$ which was correlated by the study conducted by Kamini Walia et al., by using the phenotypic method which was reported MRSA prevalence at $42 \%$ in 2008 and $40 \%$ in $2019^{(9)}$. 
Table.1 Total sample received during the study period

\begin{tabular}{|c|c|c|c|}
\hline & Positive N (\%) & Negative N (\%) & Total \\
\hline IPD & $762(20.6 \%)$ & $2,930(79.3 \%)$ & 3,692 \\
\hline OPD & $497(4.9 \%)$ & $9,450(95 \%)$ & 9,947 \\
\hline Total samples & 1,259 & 12,380 & 13,639 \\
\hline
\end{tabular}

Table.2 Sample distribution in relation of MRSA/MSSA

\begin{tabular}{|c|c|c|c|}
\hline Sample & MRSA & MSSA & Total no of Samples \\
\hline Pus & $54(81 \%)$ & $23(67 \%)$ & $77(78.6 \%)$ \\
\hline Urine & $2(\%)$ & $2(5.9 \%)$ & $4(4.1 \%)$ \\
\hline Blood & $4(6 \%)$ & $3(8.8 \%)$ & $7(7 \%)$ \\
\hline Sputum & $2(3 \%)$ & $2(5.9 \%)$ & $4(4.1 \%)$ \\
\hline Swab & $2(3 \%)$ & $2(5.9 \%)$ & $4(4.1 \%)$ \\
\hline Semen & $2(3 \%)$ & $2(5.9)$ & $2(2 \%)$ \\
\hline Total & $66(100 \%)$ & $34(100 \%)$ & $98(100 \%)$ \\
\hline
\end{tabular}

Table.3 Age distribution in relation to MRSA and MSSA

\begin{tabular}{|c|c|c|c|}
\hline Age in years & MRSA & MSSA & Total \\
\hline $\mathbf{1 - 1 0}$ & $4(11.4 \%)$ & 0 & $4(7 \%)$ \\
\hline $\mathbf{1 1 - 2 0}$ & $5(14.3 \%)$ & $5(31.2 \%)$ & $10(19 \%)$ \\
\hline $\mathbf{2 1 - 3 0}$ & $3(8.6 \%)$ & $2(12.5 \%)$ & $5(9 \%)$ \\
\hline $\mathbf{3 1 - 4 0}$ & $7(20 \%)$ & $3(18.75 \%)$ & $10(19 \%)$ \\
\hline $\mathbf{4 1 - 5 0}$ & $7(20 \%)$ & $2(18.75 \%)$ & $9(17 \%)$ \\
\hline $\mathbf{5 1 - 6 0}$ & $3(8.6 \% 0$ & $3(18.75 \%)$ & $6(11 \%)$ \\
\hline $\mathbf{6 1 - 7 0}$ & $4(11.4 \%)$ & $1(6.25 \%)$ & $5(9 \%)$ \\
\hline $\mathbf{7 1 - 8 0}$ & $2(5.7 \%)$ & 0 & $2(3 \%)$ \\
\hline$>\mathbf{8 0}$ & 0 & 0 & 0 \\
\hline Total & 35 & 16 & 52 \\
\hline
\end{tabular}

Table.4 Prevalence of Inducible Clindamycin Resistance among the isolates

\begin{tabular}{|c|c|c|c|}
\hline Variables & MRSA & MSSA & Total \\
\hline & $\mathrm{n}=40$ & $\mathrm{n}=60$ & $\mathrm{n}=100$ \\
\hline $\begin{array}{c}\text { Inducible } \\
\text { Clindamycin } \\
\text { resistance }\end{array}$ & $7(17.5 \%)$ & $2(3.3 \%)$ & \\
\hline
\end{tabular}


Fig.1 Antibiotic Susceptibility pattern

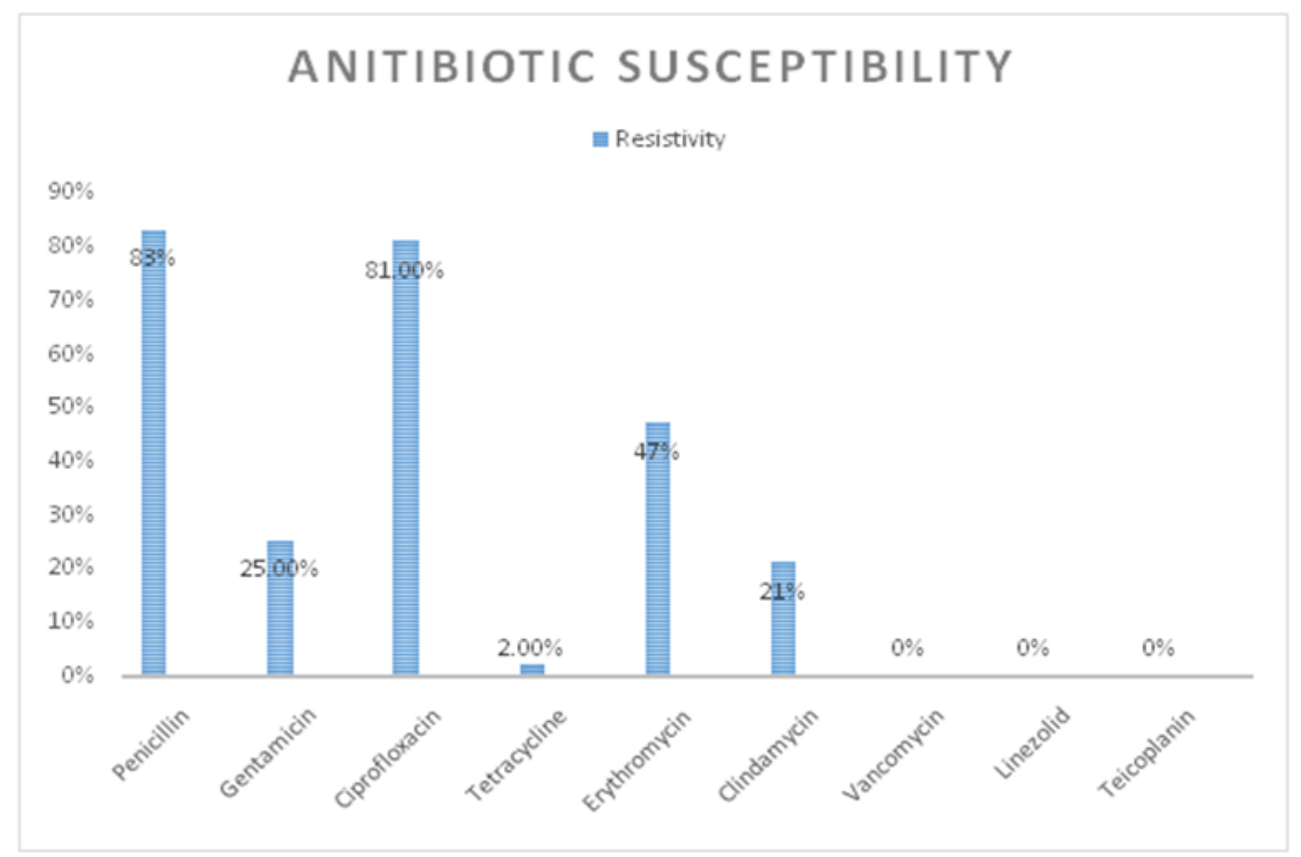

Fig.2 Prevalence of MRSA and MSSA

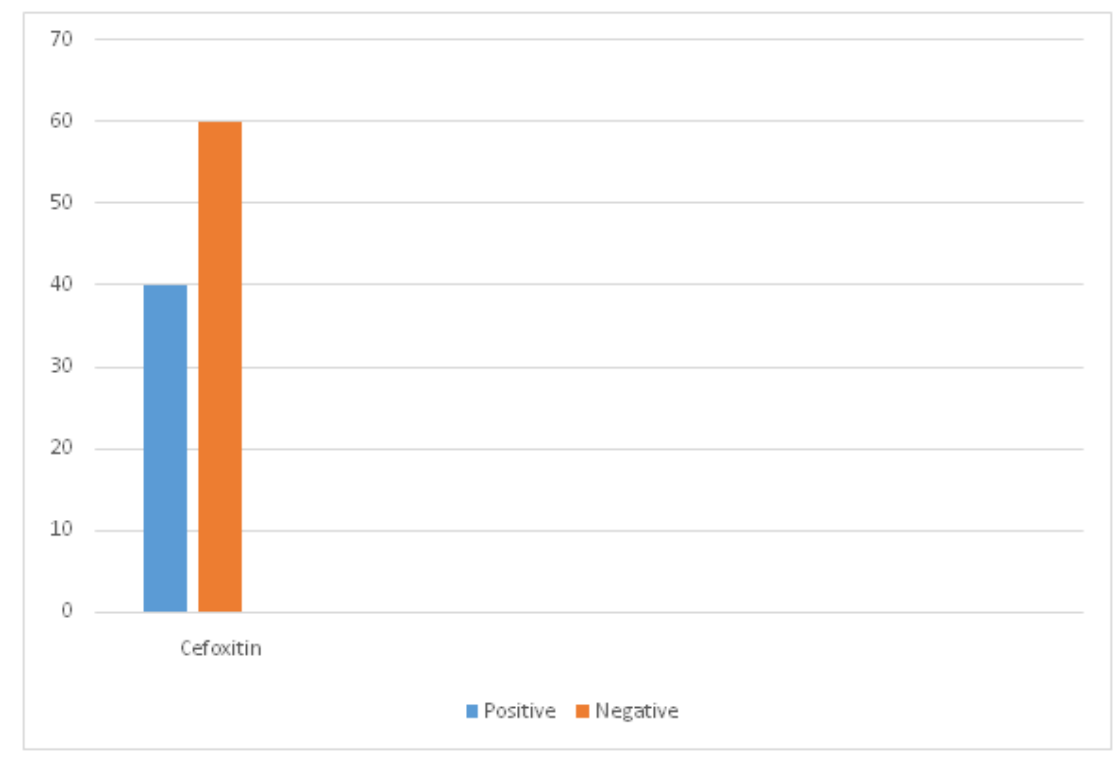

However, the study conducted by Kulkarni et al., 2014 reported very high prevalence of $\operatorname{MRSA}(70.33 \%)^{(10)}$.

In the present study, among the total of 100 isolates of Staphylococcus aureus studied, the number of isolates tested positive for
Inducible Clindamycin Resistance was 9 (9\%), of these 9 isolates, 7(17. 5\%) isolates were MRSA and 2(3.3\%) isolates were MSSA. These findings correlated well with a study conducted by the Amit Banik et al., (2015), Which showed the prevalence of inducible clindamycin resistance to be $10.7 \%$. In a study 
conducted by Lall et al., the overall prevalence of inducible clindamycin resistance among Staphylococccus aureus isolates was $20.3 \%$, and MRSA isolates exhibited $37.5 \%$ of inducible clindamycin resistance ${ }^{(11)}$. In our study the prevalence (9\%) was found to be less but higher percentage of inducible clindamycin resistance was found in MRSA $(17.5 \%)$ isolates.

Clindamycin is considered a useful alternative drug in penicillin allergic patients for treatment of skin and soft tissue infections cause by $S$. aureus. The presence of inducible clindamycin resistance among our isolates warrants routine testing to detect and initiate appropriate therapy shown in table no 4 .

In this present study, the antibiotic susceptibility of all the 100 isolates were studied by phenotypic methods. Of the 100 isolates of Staphylococcus aureus studied, $83 \%$ of the isolates were resistant to penicillin, $81 \%$ of ciprofloxacin, $71 \%$ of levofloxacin, $47 \%$ to erythromycin, $40 \%$ to cefoxitin, $25 \%$ to gentamicin, $21 \%$ to clindamycin, $2 \%$ to tetracycline. MRSA isolates showed high level of resistance to penicillin (100\%), ciprofloxacin (91\%), levofloxacin (70\%), clindamycin $(47 \%)$. None of the isolates were resistance to the vancomycin, linezolid and teicoplanin. These findings were similar to a study conducted by INSAR group (2013), which showed that among MRSA isolates $100 \%$ resistant to penicillin, $79.3 \%$ to ciprofloxacin, $70.8 \%$ to erythromycin, $46.6 \%$ to clindamycin and $58.3 \%$ to gentamicin. None of the isolates were resistant to vancomycin and linezolid ${ }^{(5)}$. A study conducted by Bhatt et al., 2014 also showed that all Staphylococcal aureus isolates were resistant to penicillin, $19 \%$ to ciprofloxacin, $40 \%$ to erythromycin, $15 \%$ to gentamicin and $14 \%$ to tetracycline. None of the isolates were resistant to vancomycin. MRSA isolates showed high level of resistant to penicillin
(100\%), ciprofloxacin (47.3\%), erythromycin $(89.4 \%)$, gentamicin $(57.8 \%)^{(12)}$ shown in fig no 1.

The present study showed the number of isolates identified as MRSA (Cefoxitin screen), were $40(40 \%)$ in number and the rest of the isolates $60(60 \%)$ were identified as MSSA. (Fig.2).

The study has shown the prevalence of MRSA that is (40\%) infections was high in our setup and is comparable to studies done earlier. Prevention of infection has to date been limited to the applications of infections control measures. The reported rate of MRSA incidence is alarming. Regular surveillance of hospital-acquired infections, isolation nursing of patients who carry MRSA, monitoring of antimicrobial susceptibility pattern, and formulation of a definite antibiotic policy may be helpful.

Our study showed high levels of resistance in MRSA isolates to various antimicrobial agents. The difficult in the therapeutic problem of multidrug resistance $S$. aureus is just one example of diminishing efficacy of antimicrobial agents for the treatment of bacterial infections.

\section{References}

1. Maslha M. Borovok I, Schreiber R et al., Analysis of transcription of the staphylococcus aureus aerobic class ib and anaerobic class III ribonucleotide reductase genes in response to oxygen. December 2001;183(24): 72607272.doi:10.1128/JB.183.24.7260-72607272.2001

2. Kluytmans J, Van Belkuma et al., Nasal carriage of Staphylococcus aureus: epidemiology, underlying mechanisms, and associated risks. July 1997;10(3):505- 
520.doi:10.1128/CMR.10.3.505-520

3. Steven Y. C Tong, Joshua S. Davis et al., Staphylococcus aureus infections: epidemiology, pathophysiology, clinical manifestations, and management. July 2015;28(3):603661.doi:10.1128/CMR.00134-14

4. Cole A M, Tahk S et al., Determinants of Staphylococcus aureus nasal carriage. November 2001;8(6):10649.doi:10.1128/CDLI.8.61064-1069

5. Tiwari H K, Sapkota D et al., High prevalence of multidrug-resistant MRSA in a tertiary care hospital of northern India. Infection and Drug Resistance. 2008; 1:57-61

6. Grundmann H, Boyce J et al., Emergence and resurgence of methicillin-resistant Staphylococcus aureus as a public-health threat; 2 september 2006:doi10.1016/S0140-6736(06)688533, p 874-885

7. Silvana $\mathrm{M} \mathrm{M}$, Emmanuel $\mathrm{R}$ et al., Prevalence of Staphylococcus aureus introduced into intensive care units of University Hospital.Dis Vol.9
8. Anupurba S, Sen M R et al., Prevalence of methicillin resistant Staphylococcus aureus in a Tertiary care Referral hospital in Eastern Utter Pradesh- IJMM 2003;21:49-51.

9. Walia $\mathrm{K}$, Madhumathi $\mathrm{J}$ et al., Establishing Antimicrobial Resistance Surveillance \& Research Network in India. 2019Feb;149(2):164179.doi:10.4103/ijmr.IJMR_226_18

10. Kulkarni S, Khare A et al., Prevalence of methicillin resistant Staphylococcocus aureus- A study in a tertiary care rural hospital; June2014:Vol-3, issue-3, P 414421

11. Lall M, Sahni A. K. Prevalence of Inducible Clindamycin Resistant Staphylococcus aureus isolated from clinical samples, Medical Journal Armed Forces India, 70,2014,43-47

12. Bhatt C. P, Karki B. M et al., Antibiotic susceptibility pattern of Staphylococcus aureus and methicillin-resistant Staphylococcus aureus in a tertiary care hospital, Journal of Pathology of Nepal (2014) Vol. 4, 548-551.

\section{How to cite this article:}

Priyanka Pradhan, Sneha Mohan, Tarana Sarwat and Dalip Kakru. 2021. Antibiogram of Staphylococcus aureus in a Tertiary Care Hospital: Changing Trends. Int.J.Curr.Microbiol.App.Sci. 10(10): 319-325. doi: https://doi.org/10.20546/ijcmas.2021.1010.040 